R. C. ALTROCK, Sacramento Peak Observatory, Sunspot, New Mexico 88349

R. G. ATHAY, High Altitude Observatory, Boulder, Colorado, 80302

K. H. BÖHM, Department of Astronomy, University of Washington, Seattle, Washington 98105

R. C. CANFIELD, Sterrewacht Sonnenborgh, Servaas Bolwerk 13, Utrecht, Netherlands

F. CAPUTO, Laboratorio di Astrofisica, Casella Postale 67, 00044 Frascati (Roma), Italia

T. R. CARSON, University Observatory, Buchanan Gardens, St. Andrews, Scotalnd, U.K.

J. M. Ie CONTEL, Observatoire de Paris, 61 Avenue de l'Observ. Paris 14e, France

Y. CUNY, Observatoire de Paris, Section d'Astrophysique, 92 Meudon, France

A. M. DELPLACE, Observatoire de Meudon, Meudon 92, France

S. DUMONT, Observatoire de Meudon, 92 Meudon, France

G. H. ELST, Sterrewacht, Ringlaan 3, Brussel 18, Belgium

H. GEROLA, Universidad de Buenos Aires, Facultad de Ciencias Exactas y Naturales, Depto de Fisica, Buenos Aires, Argentina

N. GÖKDOGAN, University obs. Astronomi kürsüsü, Beyazit, Istanbul, Turkey

G. GONCZI, Laboratoire d'Astrophysique, Faculte des Sciences, Av. Valrose, 06 Nice, France

I. P. GRANT, Atlas Computer Laboratory, Chilton, Didcot Berkshire, England

H. G. GROTH, Universitäts-Sternwarte München, 8 München 80, Scheinerstr.1, Germany

A. G. HEARN, Culham Laboratory, Abingdon, Berkshire, England

R. HERMAN, 7 bis rue Trudon, 92 Antony, France

T. HIRAYAMA, Tokyo Astr. Obs. Univ. of Tokyo, Mitaka, Tokyo, Japan

R. W. HILLENDAHL, Lockheed Palo Alto Research Laboratories 3251 Hanover Street, Palo Alto, California 94304

M. HOTINLI, University Obs. Astronomi Kürsüsü, 'Beyazit, Istanbul, Turkey

I. HOUZIAUX, Department d'Astrophysique, Faculté des Sciences, Plaine de Nimy, Mons, Belgium

D. G. HUMMER, JILA, University of Colorado, Boulder, Colorado 80302

K. HUNGER, Institut für theoretische Physik, Technische Univ. Berlin, 1 Berlin 10, ErnstReuter-Platz, Germany 
G. E. HUNT, Atlas Computer Laboratory, Chilton, Didcot, Berkshire, England

W. KALKOFEN, Harvard and Smithsonian Observatories, Cambridge, Massachusetts 02138

H. LAMERS, Sterrewacht Sonnenborgh, Servaas Bolwerk 13, Utrecht, Netherlands

A. MARTINI, Laboratorio di Astrofisica, Casella Postale 67, 00044 Frascati, Italy

CHR. MAGNAN, Institut d'Astrophysique, 98 bis BD Arago, Paris (14e), France

D. H. MENZEL, Harvard College Observatory, Cambridge, Massachusetts 02138

D. MUGGLESTONE, Department of Theoretical Physics, University of Queensland, Australia

L. NEVEN, Sterrewacht, Ringlaan 3, Brussel 18, Belgium

H. NUSSBAUMER, Physics Department, University College, Gower Street, London W.C.1., England

D. E. OSTERBROCK, Department of Astronomy, University of Wisconsin, Madison, Wisconsin

N. PANAGIA, Laboratorio di Astrofisica, Casella Postale 67, 00044 Frascati, Italy

J. C. PECKER, Observatoire de Meudon, Place Jules Janssen, 92 Meudon, France

A. PETON, ODservatoire de Meudon, Meudon 92, France

E. PEYTREMANN, Observatoire de Geneve, 1290 Sauverny, Switzerland

F. PRADERIE, Observatoịre de Meudon, 92 Meudon, France

L. PUPPI, Laboratorio di Astrofisica, Casella Postale 67,00044 Frascati, Italy

F. QUERCI, Observatoire de l'Université Toulouse, 1. Avenue Camille, Flammarion

M. RUDKJÖBING, Ole Romer Observatory, 800 Aarhus C, Denmark

G. RYBICKI, Smithsonian Astrophysical Observatory, 60 Garden Street, Cambridge, Massachusetts 02138

A. SKUMANICH, High Altitude Observatory, Boulder, Colorado 80302

D. W. STIBBS, University Observatory, Buchanan Gardens, St. Andrews, Fife, Scotland

P. SWINGS, Institut d'Astrophysique, Cointe Sclessin, Belgique

R. N. THOMAS, JILA, University of Colorado, Boulder, Colorado 80302

A. B. UNDERHILL, Sterrewacht, Servaas Bolwerk 13, Utrecht, Netherlands

R. VIOTTI, Laboratorio đi Astrofisica, Casella Postale 67, 00044 Frascati, Italy

P. WELLMANN, Universitäts-Sternwarte München 8 München 80 , Scheinerstr.1, Germany 


\section{LOCAL PARTICIPANTS}

R. Born

H. Donecker

S. Drapatz

F. Forster

K. Friedrich

M. Gumtau

R. Häfner

R. Herbst

W. Kegel

H. U. Keller

K. Kolbe

H. Kohnert

K. Metz

F. Meyer

E. Meyer-Hofmeister

G. Oleownik

H. Pfennig

G. Pöllitsch

R. Rickert

F. Schmeidler

H. Schmid

R. Schoembs

K. V. Sengbusch

M. Stix

H. C. Thomas

E. Trefftz

Ch. Wellmann

B. Wolf
USW

USW

MPI

USW

USW

USW

USW

USW

Garching, Institut Plasmaphysik

MP I

USW

USW

USW

MP I

MPI

USW

MPI

USW

USW

USW

USW

USW

MP I

MPI

MP I

MP I

USW

USW

USW = University Observatory of Munich

MPI = Max Planck Institut for Astrophysics Munich 